\title{
Incorporating Internal and External Training Load Measurements in Clinical Decision Making After ACL Reconstruction: A Clinical Commentary
}

Jeffrey B Taylor, PT, PhD, DPT, SCS, CSCS ${ }^{1}$,, Kevin R Ford, PhD, FACSM ${ }^{1}$, Robin M Queen, PhD, FACSM ${ }^{2}$, Elizabeth C Owen, DPT ${ }^{1}$, Angela Spontelli Gisselman, PT, PhD, DPT, OCS

1 Department of Physical Therapy, High Point University, ${ }^{2}$ Department of Biomedical Engineering and Mechanics, Virginia Tech

Keywords: wearable technology, return-to-sport, rehabilitation, load, acl

https://doi.org/10.26603/001c.21152

\section{International Journal of Sports Physical Therapy}

Vol. 16, Issue 2, 2021

\section{Background and Purpose}

Poor outcomes after anterior cruciate ligament reconstruction (ACLr), including the relatively high risk of suffering a subsequent ACL injury, suggest the need to optimize rehabilitation and return-to-sport testing. The purpose of this commentary is to introduce clinicians to the concept of monitoring training load during rehabilitation, to review methods of quantifying internal and external loads, and to suggest ways that these technologies can be incorporated into rehabilitation progressions and return-to-sport decisions after anterior ACLr.

\section{Description of Topic with Related Evidence}

Quantifying and identifying the effects of training load variables, external (distance, impacts, decelerations) and internal (heart rate, heart rate variability) workload, during rehabilitation can indicate both positive (improved physical, physiological, or psychological capacity) or negative (heightened risk for injury or illness) adaptations and allow for the ideal progression of exercise prescription. When used during return-to-sport testing, wearable technology can provide robust measures of movement quality, readiness, and asymmetry not identified during performance-based testing.

\section{Discussion / Relation to Clinical Practice \\ Researchers have reported the actual in-game demands of men and women of various ages and competition levels during multi-directional sport. Wearable technology can provide similar variables during rehabilitation, home exercise programs, and during on-field transition back to sport to ensure patients have met the expected fitness capacity of their sport. Additionally, clinicians can use internal load measures to objectively monitor patient's physiological responses to rehabilitation progressions and recovery rather than relying on subjective patient-reported data.}

\section{Level of Evidence}

5

\section{BACKGROUND AND PURPOSE}

Anterior cruciate ligament (ACL) tears are common injuries in cutting and jumping sports that lead to subsequent psychological and physical ramifications, including an increased risk of early onset knee osteoarthritis. ${ }^{1}$ After injury,

\footnotetext{
Corresponding Author: Jeffrey B. Taylor, PT, PhD, DPT, SCS, CSCS High Point University One University Parkway High Point, NC 27268 Phone: 1-336-841-9728 Fax: 1-336-888-5020 Email: jtaylor@highpoint.edu
} 
up to one-quarter of athletes that return to sport suffer a second ACL injury, 30\% of which occur within the first 20 athletic exposures after their return. ${ }^{2}$ These alarming statistics highlight the need to optimize rehabilitation practices and return to sport decision making.

Recovery from ACL injury involves 6-18 months of progressive rehabilitation before clearance from the medical team (i.e. surgeon, physical therapist) to return to sport. As athletes advance through rehabilitation, their progress is often tracked by the increases of repetitions, duration, intensity and the complexity of exercise. At a time when neuromuscular function (i.e. strength, coordination, functional performance) has returned to near pre-injury levels and the time since surgery ensures optimal physiologic healing of tissue, the sports medicine team (i.e. surgeon, physiotherapist, etc.) performs return-to-sport testing procedures to gauge an athlete's readiness to perform and risk of subsequent injury. ${ }^{3}$ Tests and measures that are included in these return-to-sport decisions are controversial and inconsistent, but generally include clinically-based procedures that identify residual asymmetry in strength, function, and power through isometric or isokinetic strength and singleleg hopping tests. ${ }^{3}$ Many of the current return to sport procedures are performed in controlled, clinical environments and may not elucidate an athlete's true performance or injury risk during the required demands of their sport.

Monitoring training load in an athlete's natural environment has received a lot of attention in recent literature as emerging evidence indicates a relationship between load and injury. ${ }^{4-9}$ Training load is typically categorized into either external or internal workload, with external workload capturing the mechanical work done by an athlete and internal workload referring to the physiological and psychological stress imposed by the training session on the athlete. ${ }^{10}$ Example measures of external workload include total distance travelled, the frequency of accelerations, decelerations or changes in direction, and the number of jumps performed over the course of a training or competitive session. Internal workloads can include the perception of effort in the form of ratings of perceived exertion (RPE) or physiological responses like duration of a training session spent in a specific heart rate interval, and resting heart rate variability (HRV). Quantifying and identifying the effects of these training load variables is crucial because they can indicate both positive (improved physical, physiological, or psychological capacity) or negative (heightened risk for injury or illness) adaptations. Training load measures are becoming more accessible to clinicians and coaches because they can be quantified using wearable technology or handheld devices. Instrumentation that contains global positioning system (GPS) units, accelerometers, force measuring insoles, and/or heart rate monitors are being used to quantify internal and external load variables with accuracy and precision. ${ }^{11,12}$

Workload monitoring and management has not caught on in anterior cruciate ligament reconstruction (ACLr) rehabilitation practices or return-to-sport decision making, potentially due to perceptions that measurement tools are too expensive, or analyses are too time consuming. However, workload metrics can provide valuable data for clinicians during rehabilitation procedures. While using mea- sures like repetitions and intensity can help clinicians progress individual exercises, this method fails to offer a holistic view of the training load encountered by the athlete. Likewise, current return-to-sport decision making does not factor in training load variables that may better elucidate an athlete's readiness to participate in sports with high multi-directional demands that often are not paralleled during rehabilitation sessions. ${ }^{13}$ The high rate of second ACL injury, especially within the first 20 exposures after return to sport, ${ }^{2}$ suggests that there is room for improvement in returning an athlete to sport, including current rehabilitation practices and return-to-sport decision making processes. The purpose of this commentary is to introduce clinicians to the concept of monitoring training load during rehabilitation, to review methods of quantifying internal and external loads, and to suggest ways that these technologies can be incorporated into rehabilitation progressions and return-to-sport decisions after ACLr.

\section{TRAINING LOAD MONITORING}

\section{EXTERNAL WORKLOAD}

Clinicians often monitor external workload during rehabilitation in the form of training volume, traditionally logged as a combination of the frequency (number of sets and repetitions or exercise duration) and intensity (weight or resistance) of the prescribed therapeutic exercises. ${ }^{14}$ While these traditional external workload variables are important to monitor, they are limited in scope because they do not encompass the complexity of exercise, nor do they provide a global and comprehensive view of the patient's external workload. Total training duration may give a more comprehensive view of athlete workload but does not include any measures of intensity or quality of movement that could significantly alter an athlete's response to the treatment session. To more accurately quantify external workload, clinicians may consider the use of wearable technology, such as activity monitors, accelerometers, or force measuring insoles.

\section{DISTANCE MEASUREMENTS}

Activity monitors (AM) that measure physical activity (steps per day) are gaining popularity in certain clinical populations. Clinicians and researchers have used AM in various ways, including assessing the effectiveness of cardiac rehabilitation 15 and other behavioral interventions. ${ }^{16}$ Additionally, AM have been used to quantify activity in populations such as children with cerebral palsy ${ }^{17}$ and adults after stroke, ${ }^{18}$ and have been used in rehabilitation settings like skilled nursing facilities and inpatient rehabilitation to gauge the intensity of exercises and patient participation during treatment sessions. ${ }^{19,20}$ AM vary depending on the manufacturer and model but are often worn at waistlevel or on the distal aspect of the arm or leg. These devices combine data from an embedded tri-axial accelerometer with a proprietary algorithm to measure step counts and calculate distance as a function of step count and step length. Off-the-shelf AM (i.e. FitBit) are generally reliable for tracking step counts and distances. ${ }^{21,22}$ Research- and clinical-grade AM instrumentation (i.e. ActiGraph) can pro- 
vide more reliable spatiotemporal variables than commercially-available AM during both walking and running, ${ }^{23}$ including step counts and distances and the duration of any bouts of active or sedentary time when worn for an extended period. Though a few studies have reported using AM to measure physical activity in youth sports, ${ }^{24,25}$ the use of AM has yet to gain traction in sports medicine settings because of time and cost perceptions.

Global positioning systems (GPS) are becoming more common in team sports, especially for tracking distances traveled by players during practices and games..$^{11}$ Since GPS technology can accurately pinpoint one's location using a series of orbiting satellites, GPS units calculate distances travelled based on changes in location. Activity monitors and/or GPS units can be a cost-effective complement to traditional external workload measures during rehabilitation and return-to-sport after ACLr. There is emerging evidence reporting average sport-specific loads that can provide guidelines to prepare athletes to return to their sport. For example, elite soccer players travel an average of over 10,000 meters, whereas basketball players travel 6,000-7,000 meters per contest. ${ }^{9}$ Clinicians should ensure that athletes have built the requisite fitness base and have received adequate exposure to similarly matched load intensities for safe return to their sport. Most published data are based on game data, but sport practices have the potential for greater intensity and larger distances traveled, which may need to be accounted for in rehabilitation. Not quantifying rehabilitation sessions or monitoring an athlete outside of the rehabilitation setting could result in the clearance of an athlete for return to sport from strength and symmetry measures without evidence that the athlete is prepared to tolerate the expected workload when released to compete. This could happen in a traditional rehabilitation setting, or with an independent strength and conditioning professional. Regardless, the clinician that ultimately makes the return to sport decision needs to be knowledgeable of these data. In addition to providing valuable information for clinicians, AM or GPS units can also be used to help with exercise prescription outside of the clinical setting. It may be unrealistic for an athlete to simulate $10,000+$ meters in a rehabilitation setting; however, using this wearable technology, clinicians can prescribe progressive distances as part of a home exercise program. Traditionally, this is done with straight line running distance because of the ease of measurement, but most sports require multi-directional movements that can be quantified in realtime with AM or GPS units.

EXAMPLE APPLICATION DURING REHABILITATION AND RETURN-TO-SPORT TESTING

Figure 1 depicts an ideal theoretical rehabilitation management model for an athlete after ACLr. This model should be individualized to the athlete and could be uni- or multivariate, depending on the sport's activity demands. For this example, consider total distance covered as measured by an activity monitor. When monitoring these variables after ACLr, clinicians should account for both dedicated rehabilitation workload (whether performed in a clinical setting or as a home exercise program) and other physical activity. When progressing distance workload, clinicians must rely on their experience, physiological healing of the tissue, the patient's response to treatment (potentially including internal load measures as described later in this review), and the patient's goals (including sport-specific demands). The model shows a total daily distance spike (time of rapid increase in distance) around two-weeks post-operative when gait and functional workload begin to normalize and pain decreases, and around 12-weeks which is the typical time of initiating a jogging/running program post-ACLr if certain criterion are met. ${ }^{26-28}$ Similarly, the model shows a distance spike in dedicated rehabilitation distance around week 12 . There is then a steady progression of distance during rehabilitation to reach average and maximal sport demands, though the pace of progression slows to stabilize the acute-to-chronic workload ratio. Monitoring these variables elucidates the athlete's fitness capacity for clinicians and can be used as comparisons to previously published values specific to the patient's age, sex, competitive level, and sport during the return-to-sport decision making process. The goal is that the athlete has the capacity to load at average and maximal game demands before being fully cleared to return-to-sport. While research is needed to validate this model, it helps illustrate the potential interaction of daily and rehabilitation workload and shows how to incorporate activity demands as specific and meaningful long-term goals during rehabilitation progressions.

\section{GROUND REACTION FORCE}

Ground reaction forces can help clinicians quantify magnitudes and asymmetries of limb loading. In laboratory settings, force platforms can provide 3D analysis of ground reaction forces but are expensive and not accessible to most clinicians. Force measuring insoles (i.e. loadsol ${ }^{\circledR}$, Novel Electronics, St. Paul, MN) can provide valid and reliable asymmetry data during squatting, running, and landing. ${ }^{29-31}$ Force measures using portable insoles have been reported to be highly correlated with knee extension moment asymmetries during landing, ${ }^{31}$ a common finding in individuals after ACLr that may be predictive of a subsequent injury. ${ }^{32}$ In addition to their prognostic value, these data can be used throughout the rehabilitation process to provide biofeedback to patients to improve neuromuscular control (i.e. increase or reduce forces) and normalize movement between the reconstructed and unaffected limbs. The assessment of limb loading asymmetry can be a valuable contribution during both early-stage rehabilitation as the athlete attempts to regain symmetry with controlled movements like gait and squatting, and in late-stage rehabilitation when determining return-to-sport readiness. Using force measuring insoles during return-to-sport testing allows clinicians to assess movement quality differences between the reconstructed limb and healthy limb. ${ }^{33}$ Accelerometers measuring tibial acceleration can also be used as a surrogate measure for ground impact and can be easily used in controlled clinical or natural playing environments. ${ }^{34}$

EXAMPLE APPLICATION DURING REHABILITATION AND RETURN-TO-SPORT TESTING

During early post-operative rehabilitation, a patient's 


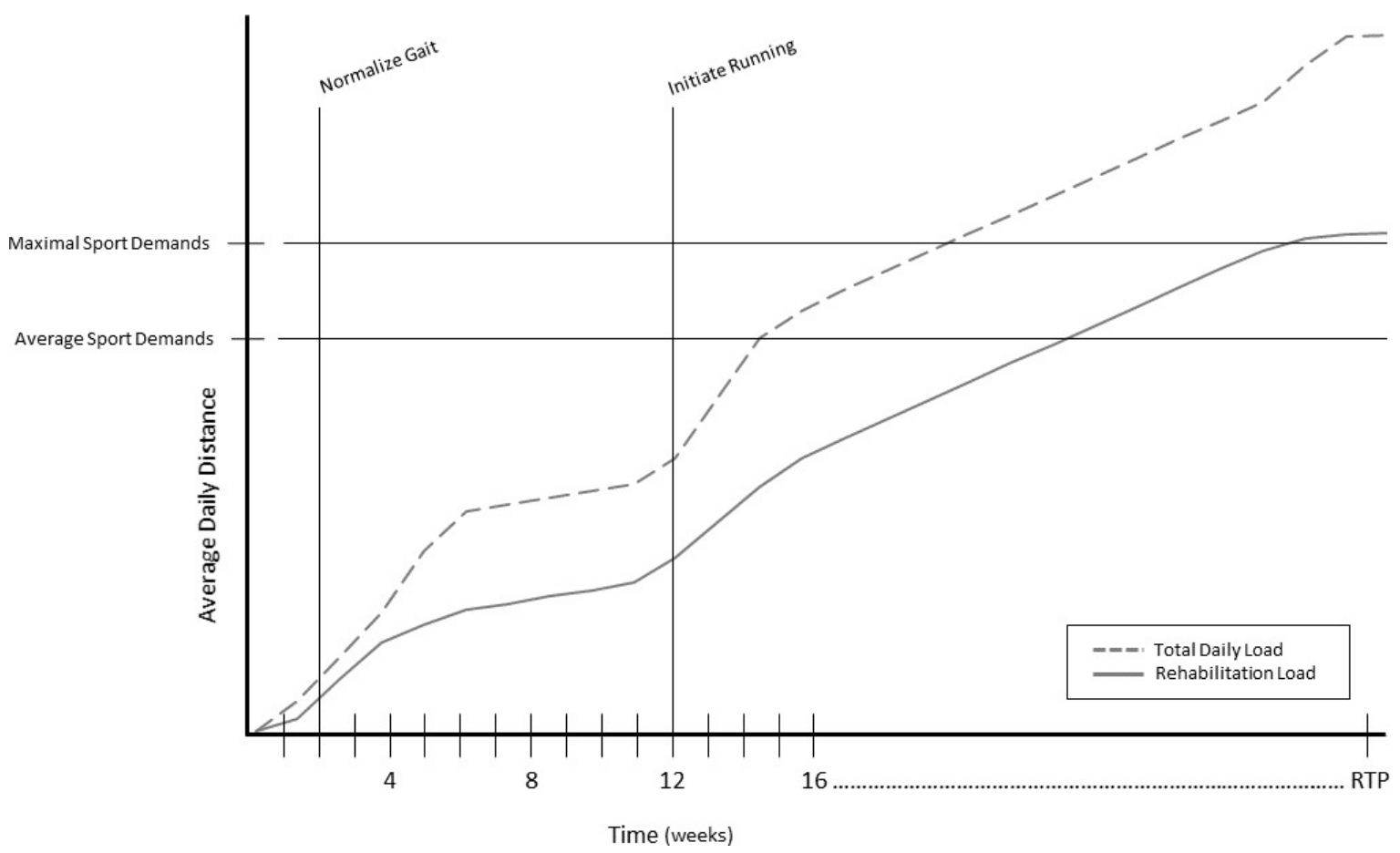

Figure 1: Theoretical progression of an athlete's total distance travelled after ACLr.

ground reaction force can be displayed during a variety of two-legged squatting progressions or other activities. This biofeedback allows the patient to correct asymmetry in real-time using an external focus. ${ }^{35}$ The goal in the earlyphase would be to regain symmetry by weight-shifting towards the reconstructed limb. During later-stage rehabilitation, clinicians may use a similar approach to overload the reconstructed limb for preparation of asymmetries that would occur during sport.

Ground reaction forces can also be utilized during singleleg activities both during rehabilitation and return-to-sport testing. Figure 2 shows the vertical ground reaction force curves during gait of a patient after ACLr at post-operative weeks 12 and 31. At week 12, impact peaks and loading rates at heel strike appear relatively symmetrical, yet the patient produces significantly less ground reaction force during push off in the reconstructed limb. At week 31, the patient is loading the reconstructed limb at higher levels than the non-injured limb. While other contextual factors should be accounted for, alone these graphs show a significant positive progression in the patient's confidence in and function of the reconstructed limb.

\section{MULTI-DIRECTIONAL MOVEMENTS}

Deceleration is a primary mechanism of lower extremity injury and non-contact ACL rupture. ${ }^{36}$ A vast majority of ACL injuries occur during decelerations associated with cutting (horizontal change in direction) and landing (vertical change in direction). ${ }^{37}$ During rehabilitation, clinicians must simulate sport-specific game demands and emphasize both the quality (movement patterns) and quantity (frequency) of accelerations and decelerations before returning to play.
Cutting is a necessary component of a rehabilitation plan for patients' that participate in sports like soccer, which demands up to 700 cuts per game. ${ }^{9}$ An accelerometer can measure the quantity and intensity of simulated cuts during rehabilitation, home exercise programs, and return-tosport testing procedures. Current clinical tests of agility provide time measures for the completion of a test, but to the authors' knowledge, there are no clinically accessible agility measures that identify asymmetries, and thus may not fit in the return-to-sport testing battery. Being able to quantify accelerations and decelerations during procedures like the T-test (Figure 3) ${ }^{38}$ pro-agility, ${ }^{39}$ and lower extremity functional test (LEFT), ${ }^{40}$ may provide crucial information about an athlete's ability to create power and speed on an individual limb during a functional activity.

Like cutting, jumping and landing require repetitive demands (> 50 jumps per volleyball and basketball match), making it essential to monitor jump frequency and landing force in athletes rehabilitating from ACLr. ${ }^{9}$ Commercial accelerometers worn at waist-level can track an athlete's jump count, jump height, and landing. Much like total distance and cutting, clinicians should have an appreciation for the sport-specific demands of their athlete upon return to sport and ensure that their rehabilitation plan adequately prepares the athlete for safe return. Jump count may not be as critically under-prescribed as others measures during rehabilitation because of the relatively common use of plyometric activities in ACL rehab programs $;{ }^{41,42}$ however, these plyometric programs need to be implemented and progressed in a safe manner. Accelerometers that record jump count can be used to monitor and progress plyometric training loads during rehabilitation (e.g. VERT wearable jump monitor, Mayfonk Athletic, Fort Lauderdale, FL). ${ }^{43}$ Realtime jump height measurements can also be a valuable per- 

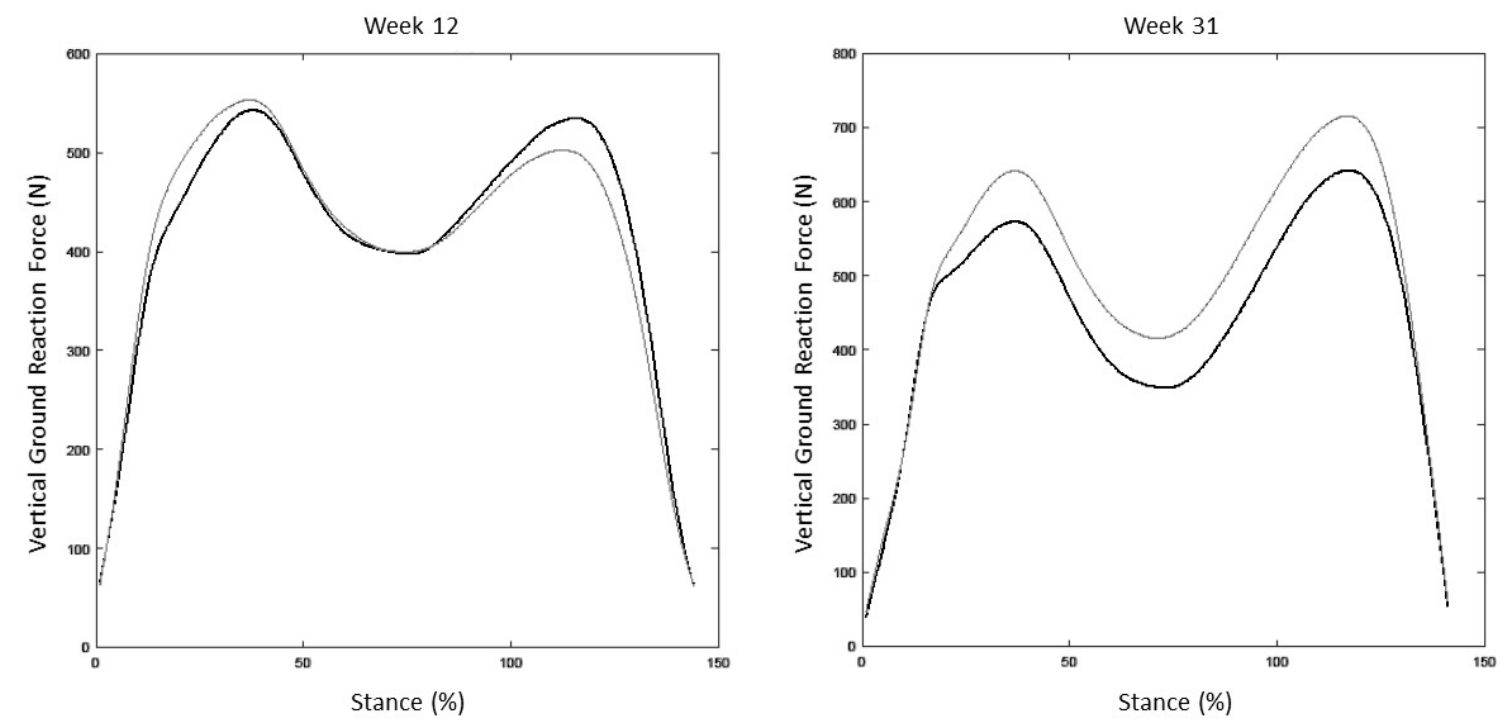

Figure 2: Example vertical ground reaction curves during gait of a patient after ACLr at post-operative week 12 and week 31 .

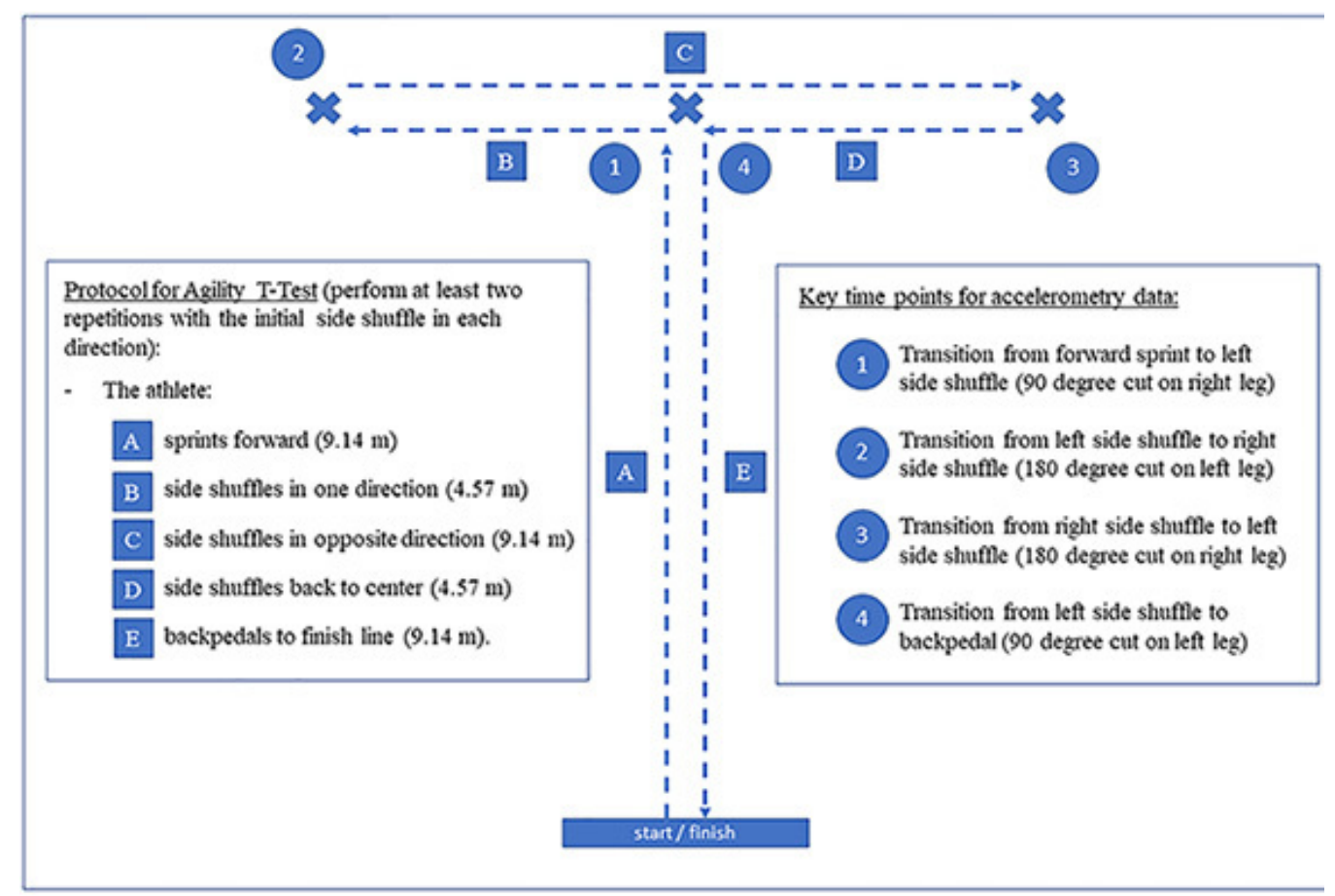

Figure 3: Example use of accelerometry during agility t-test.

formance variable to monitor during rehabilitation. Jump height can help quantify the intensity of an exercise during an individual rehabilitation session or ensure that an athlete's performance progresses appropriately during early stages of rehabilitation and does not decline as other load factors increase in preparation for return-to-sport.
EXAMPLE APPLICATION DURING REHABILITATION AND RETURN-TO-SPORT TESTING

Figure 4 shows the progression of accelerometer measured jump count throughout post-operative rehabilitation for an adolescent, competitive, female soccer player. ${ }^{13}$ Similar to progressing distance workloads in rehabilitation, clinicians 


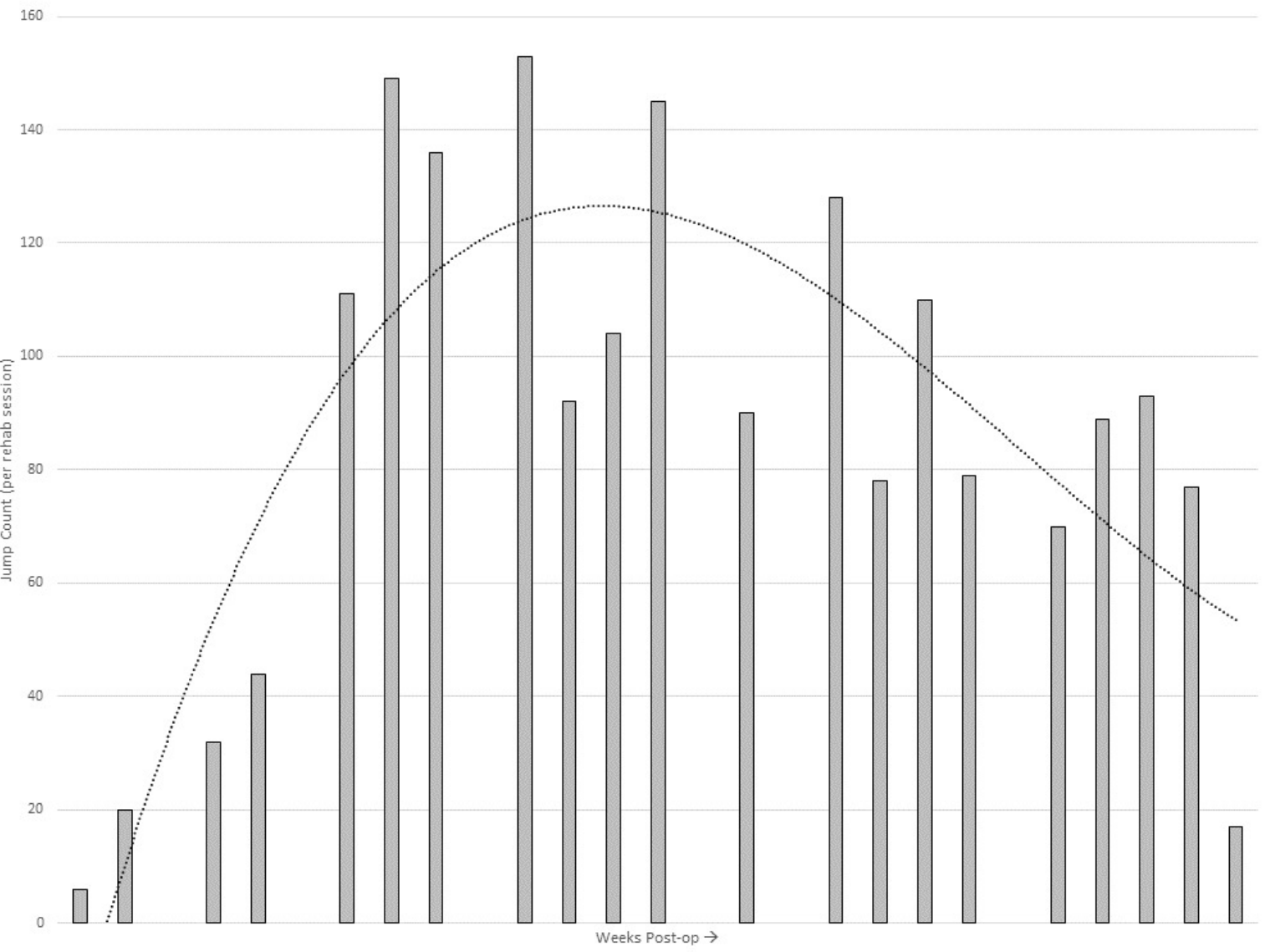

Figure 4: Example progression of jump count during post-operative rehabilitation of a patient after ACL reconstruction (Used with permission of The International Journal of Sports Physical Therapy. Taylor JB, Owen E, Ford KR. Incorporating workload measures into rehabilitation after anterior cruciate ligament reconstruction: A case report. Int J Sports Phys Ther. 2020;15(5):823-831.)

must rely on their experience, physiological healing of the tissue, the patient's response to treatment, and the patient's goals. In this example, jump count shows a substantial increase throughout weeks $13-23$ as the patient participates in progressive plyometric training to improve strength, power, speed and athletic function. Then, knowing that the patient's goal is to return to soccer, exercise prescription transitions away from jumping to cutting and other horizontal agility exercises and drills to match the demands of soccer. An athlete that participates in a more jump-dominant sport like basketball or volleyball may benefit from a more sustained frequency level of high jumps, or in the case of a volleyball setter may need to increase towards $>200$ submaximal jumps to meet the demands of the sport. ${ }^{44}$

\section{INTERNAL LOAD}

A holistic consideration of training load in rehabilitation progressions should include measures of both external and internal stressors. ${ }^{10,45}$ Common factors that contribute to the overall physical, physiological, and psychological loading on the system include elements such as travel, academic demands, non-sport stressors, sleep, nutrition, and subjective wellbeing. ${ }^{10}$ Although it is impossible for a single measure to quantify the interactions of numerous variables on an athlete's adaptation to load, monitoring internal workload can complement current ACLr rehabilitation procedures. ${ }^{7,10,46}$

\section{RATING OF PERCEIVED EXERTION}

There are numerous non-invasive, time-effective options for quantifying internal load that are frequently used in athlete monitoring and could help guide the rehabilitation process. Session RPE (SRPE) is a cost and time effective option that measures an athlete's perceived intensity of a training session. Immediately following a training session, the athlete provides a subjective report of the intensity of the session using a 10-item Borg's CR10 scale. This number is multiplied by the total minutes of the training session to yield SRPE. ${ }^{47,48}$ sRPE has been strongly correlated with heart rate during steady state and high intensity exercise. ${ }^{47-49}$ In addition to its correlation with cardiorespiratory fitness, SRPE, when monitored and analyzed over time, demonstrates some promise as a predictor of injury 
when combined with measures of an athlete's well-being. 50 A common criticism of SRPE is that this number may not accurately represent the intensity of a training or rehabilitation session. For example, when an athlete records a total daily training load of 180 arbitrary units, this value could indicate a near-maximal training session (RPE $=9$ ) that was 20 minutes in duration or, an easy training session $(\mathrm{RPE}=2)$ that was 90 minutes in duration. While it is clear that SRPE is influenced by subjectivity, this load monitoring method is considered a valid tool for assessing an athlete's physiological response to training. ${ }^{47-49}$ Though challenging in the traditional clinical setting, sRPE should ideally be recorded 20-30 minutes after training for adequate reflection time and to avoid recency bias. ${ }^{51}$ If not taken the day of a session, sRPE may also be collected before a training session to gauge the level of intensity of the previous session and help determine the intensity of the upcoming session.

Another low-cost option for monitoring athletes' tolerance to training and non-training stressors are wellness and/or psychological questionnaires. There are a multitude of questionnaires available, some are specific to sport (e.g. Acute Recovery and Stress Scale), while others monitor general well-being (e.g. Profile of Mood States, POMS), or measure across both domains (e.g. Recovery-Stress Questionnaire for Athletes, REST-Q-Sport and Recovery-Cue). Clinicians must attempt to avoid common barriers to success including inadequate buy-in from all stakeholders (e.g. the athlete, coaches, rehabilitation team), inconvenient and/or inconsistent methods of data collection, and the lack of use of data gathered from the questionnaire for training modification purposes. 52

EXAMPLE APPLICATION DURING REHABILITATION AND RETURN-TO-SPORT TESTING

As a monitoring tool that requires minimal effort or advanced technology to record, clinician's regular use of SRPE after each treatment session can provide valuable information that subjective pain scores may not capture. To easily capture SRPE, clinicians can quickly record the duration of a rehabilitation session and the athletes' subjective report of its intensity in medical documentation. A sudden change in the athlete's perceived intensity of a rehabilitation session that was designed to gradually, and not suddenly, challenge the athlete would be revealed when collecting sRPE. This type of information may not always be reflected when assessing post-treatment pain levels. In turn, this data allows the clinician to engage in a meaningful and holistic conversation with the athlete to investigate other sources of potential stressors (e.g. poor sleep habits, academic stressors, travel, anxiety, fear of movement, etc.) that may be contributing to the athlete's reports of increased intensity of a rehabilitation session. Additionally, clinicians can use sRPE as a method to assess whether their treatment sessions are achieving the desired intensity at each stage along the ACLr rehabilitation timeline, and especially near the end of rehabilitation when intensity should rise significantly to match the demands of return to sport play.

\section{HEART RATE AND HEART RATE VARIABILITY}

Heart rate (HR) has been used to monitor the influence of exercise load on the body. ${ }^{53-58}$ HR can quantify the body's tolerance to external stressors (e.g. exercise). When measured at rest, during, or immediately following exercise, HR measures may represent autonomic nervous system (ANS) control of the heart and provide sensitive indicators of the body's response to exercise load. $55,59,59,60,60$

A critical aspect of rehabilitation is delivering the appropriate dose of an intervention to progress a patient towards targeted outcomes. While dosage is most frequently adjusted based on patient response (e.g. reported pain levels, changes in range of motion or movement pattern after a selected intervention), resting HR data can assist clinicians in providing more individualized intervention prescription. When monitored consistently over time, patterns in resting HR and heart rate variability (HRV) can reflect an athlete's physiological and psychological adaptation to and readiness for rehabilitation and other life stressors that influence a patient's trajectory to recovery (e.g. fatigue, sleep quality).

HRV is a measure that has attracted the attention of sport scientists, coaches, and clinicians due to its sensitivity as an index of athletes' physiological response to load. ${ }^{60-63}$ HRV is derived from the variations in time between the R$\mathrm{R}$ intervals identified on an electrocardiogram trace (Fig$\underline{\text { ure 5). HRV represents the synchronization of the ANS }}{ }^{64-66}$ and is an indirect measure of the dynamic coordination between the sympathetic and parasympathetic systems on the heart during stress and recovery. ${ }^{60,67,68}$ Resting HRV is predominantly controlled by parasympathetic, or vagal, tone and this has been previously confirmed by pharmacological blockade studies. ${ }^{69}$ HRV can provide a sensitive and accurate reflection of the body's physiological response to load, and therefore is an important measure to consider alongside rehabilitation protocols. ${ }^{59}$

When assessed at the beginning of a training session, HRV can serve as a physiological readiness index with minimal resource burden and high potential to inform training load management methods for improved sport performance outcomes. HRV can be measured non-invasively and quickly (<60 seconds) with electrocardiogram, chest strap HR monitors or smartphone-based technologies. ${ }^{70}$ With technological advancements such as ring-based (e.g. Ōura Ring, Oura, Oulu, Finland) and smartphone-based photoplethysmogram (PPG) acquisition, PPG-based HR monitoring is readily available and proving to be just as valid and reliable as heart rate strap measurements. ${ }^{60,61,71-74}$

Recent investigations using HRV as an internal load measure demonstrate strong associations between changes in HRV and external training load. 55,75 Athletes responding positively to training demonstrate increased HRV, reflecting an optimal functioning ANS. ${ }^{59}$ An organism expresses homeostasis through the adaptability and variability of the systems responding to stressors and maintaining overall health. In the case of the ANS, optimal homeostasis is represented by increased parasympathetic modulation at the heart. ${ }^{76}$ Rapid adjustments in heart rate can be made under increased parasympathetic control, allowing the body greater adaptability when responding to stressors or stimuli. ${ }^{66,77}$ Increased parasympathetic activity results in lowered average heart rate and an increased variability of the time interval between each heartbeat (that is, an increase 


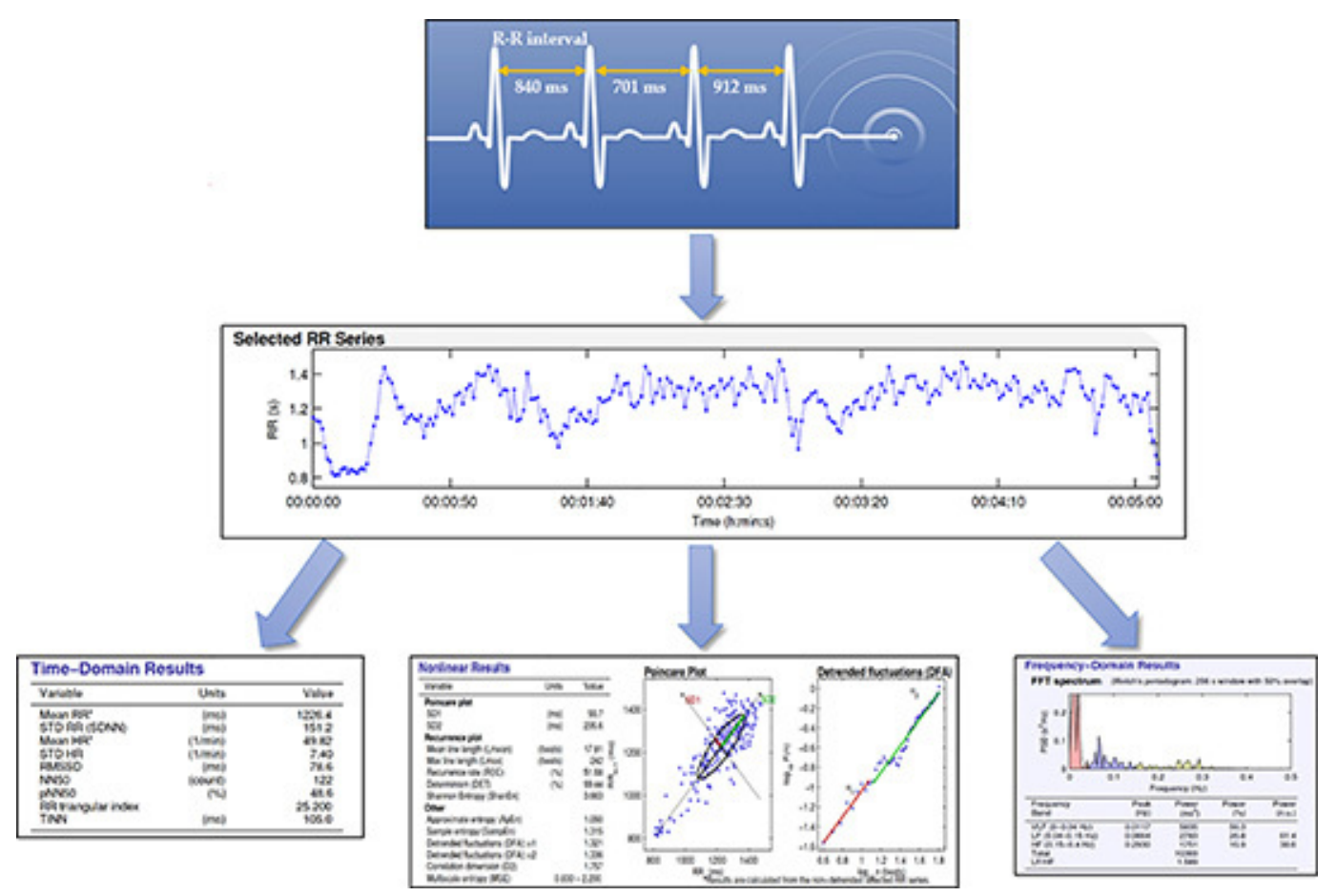

Figure 5: Heart rate variability analysis. After proper identification of RR intervals from electrocardiogram trace (top picture), the measured time of each RR interval is plotted over time (middle picture). From the selected RR series, three common methods of HRV analysis can be performed: time domain, nonlinear and frequency domain (three bottom pictures).

in HRV measures). ${ }^{76}$ Said another way, increased HRV represents greater parasympathetic modulation at the heart, and reflects an adaptable and healthy ANS that can respond quickly to stressors such as exercise or strenuous training.

Conversely, reductions in HRV can be indicative of less parasympathetic modulation at the heart and in some cases, increased sympathetic nervous system control. This imbalance of the ANS is reflected in heightened average heart rate, which leads to decreased variability of the time interval between each heartbeat and decreased HRV measures. Reductions in resting HRV have been researched as a physiologic marker of negative consequences in athletes diagnosed with overtraining syndrome who demonstrate long-term decrements in performance along with physiological and/or psychological signs and symptoms that resemble disturbed ANS function (e.g. excessive fatigue, altered sleep, depression). ${ }^{78}$ The uses of HRV in sport performance provide pragmatic examples of how monitoring patterns of change in a surrogate health measure like HRV can inform our understanding of risk profiles for overloading or perhaps underloading our athletes. Studying HRV patterns in response to rehabilitation and other nontraining stressors provides useful information related to how the function of the ANS influences recovery after loading of somatic tissues. ${ }^{79}$

EXAMPLE APPLICATION DURING REHABILITATION AND RETURN-TO-SPORT TESTING

Similar to how HRV has been used to successfully design training programs to maximize performance and avoid excessive fatigue or overtraining, ${ }^{80,81}$ information gained from HRV may guide rehabilitation progressions, in order to protect and enhance the recovery of injured tissues. Theoretically, HRV could serve as a "vital sign" in rehabilitation whereby clinicians receive daily HRV recordings and are alerted when there are significant pattern changes. Since ANS also holds a commanding response to stressors such as illness or injury, increases in weekly HRV readings typically signify that a patient is responding well to stressors such as exercise intervention and other non-therapy related stressors (e.g. sleep quality and fatigue). Alternatively, significant decrements in weekly HRV readings may indicate an overloaded ANS and therefore adjustments in the patient's plan of care can be made accordingly. This hypothesized scenario would improve accuracy of 'reloading' as a part of rehabilitation, which may allow clinicians and coaches to modify the negative impact of pertinent injury risk factors (e.g. fatigue, poor sleep quality, inadequate nutrition) on an athlete's rehabilitation or return to sport progression.

\section{SUMMARY AND CONCLUSIONS}

Most ACL rehabilitation protocols and guidelines have shifted from time-based to criterion-based progressions to allow for individualization of rehabilitation and may help reduce the risk of under- or over-loading. The authors suggest adding parameters to these guidelines that incorporate internal and external workload measurements. For example, using normative sport demands as goals or thresholds during rehabilitation may help reduce under-loading that may occur during rehabilitation and ensure that the athlete has built up the requisite fitness to return to their sport. ${ }^{9}$ 


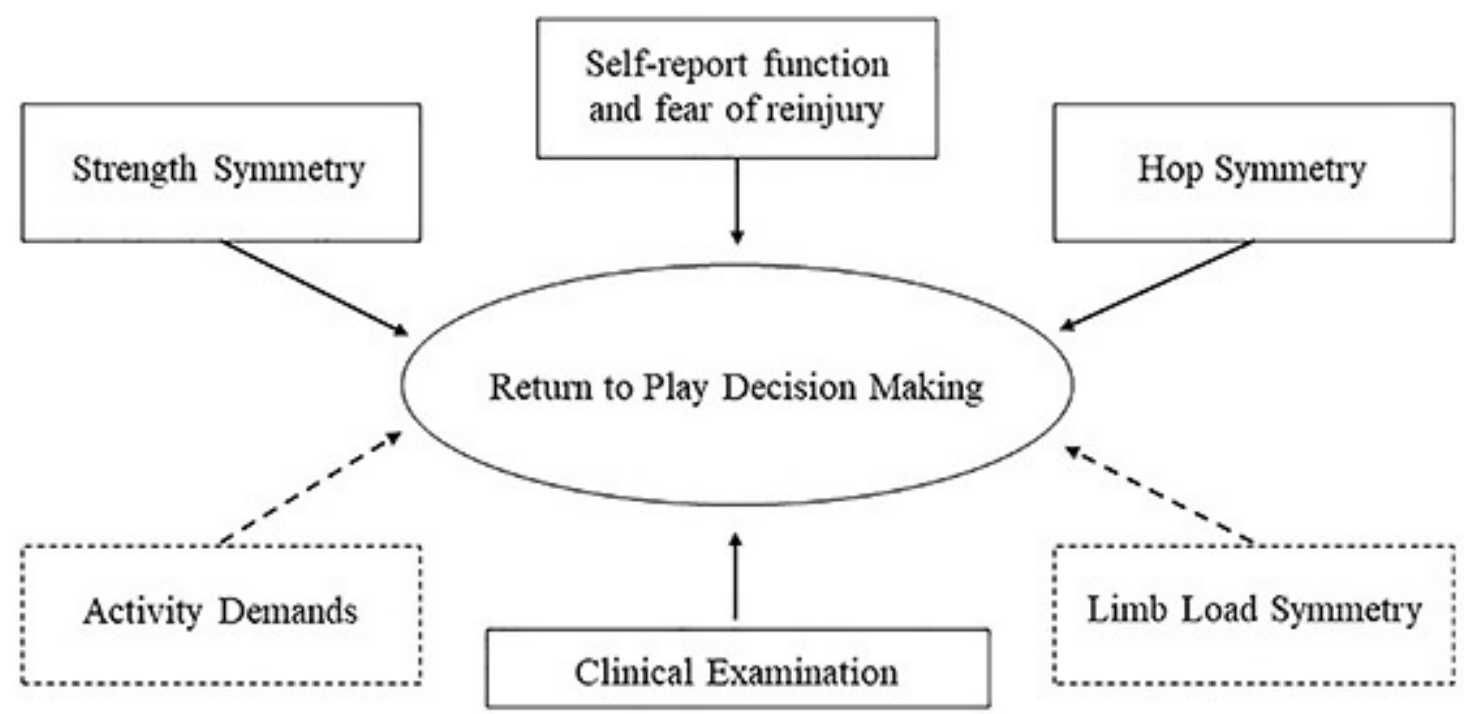

Figure 6: Current factors (solid line) and factors newly available to easily measure (dotted line) that may affect the return-to-sport decision making process after ACLr.

Further, close monitoring of variables like distance and jump count can promote more systematic exercise progressions and may decrease the risk of injury (e.g. patellar tendinopathy) from repetitive overuse or under-recovery. ${ }^{42}$

While monitoring and progressing external workload during rehabilitation, internal workload can help clinicians justify their rate of progression. The application of improved research methodologies in recent studies is revealing increasingly consistent directional changes of parasympathetic-mediated markers of HRV in response to athletes' positive adaptations to training. ${ }^{82}$ Taken together, this evidence supports the previously-established hypothesis that, in general, athletes who exhibit an increase in parasympathetic-mediated HRV measures in response to training load are coping positively, performing optimally in their sport, and perhaps are at less risk of injury. ${ }^{59,75,80,82}$

During return-to-sport testing and decision making, current best practice utilizes a combination of clinical examination (pain, effusion, laxity), single-leg hop symmetry tests, single-leg isokinetic strength symmetry tests, and functional or psychological self-report questionnaires to gauge an athlete's readiness to return-to-sport. ${ }^{81}$ Other measures of external workload, including activity demands and limb loading symmetry during sport-specific activities may help complement the aforementioned testing battery (Figure 6). Athletes need to have the requisite physiologic readiness levels to safely return to sport. Clinicians can now monitor their athletes' activity demands to ensure they are at a level commensurate with their sport. Using wearable technology, clinicians can gather robust information on limb loading symmetry. Forces that occur during sportspecific movements like landing and cutting represent movement quality measures that may not be perceived without technology. After ACLr, athletes should not be allowed to return to their sport unless the athlete is able to withstand external and internal workloads without deterioration of biomechanics or significant fatigue.

Measures of external and internal workload may provide important complementary information to clinicians involved in any stage of rehabilitation or the return to sport decision making process after ACL reconstruction. Wearable technologies are becoming increasingly more accessible, both in terms of cost and ease of use, and the data they provide can help quantify rehabilitation progressions, serve as comparisons for established sport-specific workload demands, and identify the athlete's physiological well-being.

\section{FUNDING / CONFLICTS OF INTEREST}

No funding was received for this study. The authors declare no conflicts of interest.

Submitted: May 20, 2020 CDT, Accepted: October 10, 2020 CDT 
This is an open-access article distributed under the terms of the Creative Commons Attribution 4.0 International License (CCBY-NC-SA-4.0). View this license's legal deed at https://creativecommons.org/licenses/by-nc-sa/4.0 and legal code at https://creativecommons.org/licenses/by-nc-sa/4.0/legalcode for more information. 


\section{REFERENCES}

1. Luc B, Gribble PA, Pietrosimone BG. Osteoarthritis prevalence following anterior cruciate ligament reconstruction: a systematic review and numbersneeded-to-treat analysis. J Athl Train. 2014;49(6):806-819. doi:10.4085/1062-6050-49.3.35

2. Paterno MV, Rauh MJ, Schmitt LC, Ford KR, Hewett TE. Incidence of second acl injuries 2 years after primary acl reconstruction and return to sport. Am J Sports Med. 2014;42(7):1567-1573. doi:10.1177/03635 46514530088

3. van Melick N, van Cingel RE, Brooijmans F, et al. Evidence-based clinical practice update: practice guidelines for anterior cruciate ligament rehabilitation based on a systematic review and multidisciplinary consensus. Br J Sports Med. 2016;50(24):1506-1515. doi:10.1136/bjsports-2015-09 $\underline{5898}$

4. Drew MK, Finch CF. The relationship between training load and injury, illness and soreness: A systematic and literature review. Sports Med. 2016;46(6):861-883. doi:10.1007/s40279-015-0459-8

5. Vanrenterghem J, Nedergaard NJ, Robinson MA, Drust B. Training load monitoring in team sports: A novel framework separating physiological and biomechanical load-adaptation pathways. Sports Med. 2017;47(11):2135-2142. doi:10.1007/s40279-017-071 $\underline{4-2}$

6. Jaspers A, Kuyvenhoven JP, Staes F, Frencken WGP, Helsen WF, Brink MS. Examination of the external and internal load indicators' association with overuse injuries in professional soccer players. J Sci Med Sport. 2018;21(6):579-585. doi:10.1016/j.jsams.2017.10.005

7. Bourdon PC, Cardinale M, Murray A, et al. Monitoring athlete training loads: Consensus statement. Int J Sports Physiol Perform. 2017;12(Suppl 2):S2161-S2170. doi:10.1123/IJSPP.2017-0208

8. Cardinale M, Varley MC. Wearable trainingmonitoring technology: Applications, challenges, and opportunities. Int J Sports Physiol Perform. 2017;12(Suppl 2):S255-S262. doi:10.1123/ijspp.201 $\underline{6-0423}$

9. Taylor JB, Wright AA, Dischiavi SL, Townsend MA, Marmon AR. Activity demands during multidirectional team sports: A systematic review. Sports Med. 2017;47(12):2533-2551. doi:10.1007/s40279-01 7-0772-5
10. Soligard T, Schwellnus M, Alonso JM, et al. How much is too much? (Part 1) International Olympic Committee consensus statement on load in sport and risk of injury. Br J Sports Med. 2016;50(17):1030-1041. doi:10.1136/bjsports-2016-096581

11. Scott MT, Scott TJ, Kelly VG. The validity and reliability of global positioning systems in team sport: A brief review. J Strength Cond Res. 2016;30(5):1470-1490. doi:10.1519/JSC.00000000000 $\underline{01221}$

12. O’Driscoll R, Turicchi J, Beaulieu K, et al. How well do activity monitors estimate energy expenditure? A systematic review and meta-analysis of the validity of current technologies. Br J Sports Med. 2020;54(6):332-340. doi:10.1136/bjsports-2018-09964 $\underline{3}$

13. Taylor JB, Owen EC, Ford KR. Incorporating workload measures into rehabilitation after anterior cruciate ligament reconstruction: a case report. Int J Sports Phys Ther. 2020:in press.

14. Kraemer WJ. Strength training basics: Designing workouts to meet patients' goals. Phys Sportsmed. 2003;31(8):39-45. doi:10.3810/psm.2003.08.457

15. Prince SA, Blanchard CM, Grace SL, Reid RD. Objectively-measured sedentary time and its association with markers of cardiometabolic health and fitness among cardiac rehabilitation graduates. Eur J Prev Cardiol. 2016;23(8):818-825. doi:10.1177/20 $\underline{47487315617101}$

16. Geidl W, Semrau J, Streber R, et al. Effects of a brief, pedometer-based behavioral intervention for individuals with COPD during inpatient pulmonary rehabilitation on 6-week and 6-month objectively measured physical activity: study protocol for a randomized controlled trial. Trials. 2017;18(1):396. $\underline{\mathrm{d}}$ oi:10.1186/s13063-017-2124-Z

17. O’Neil ME, Fragala-Pinkham MA, Forman JL, Trost SG. Measuring reliability and validity of the ActiGraph GT3X accelerometer for children with cerebral palsy: a feasibility study. J Pediatr Rehabil Med. 2014;7(3):233-240. doi:10.3233/PRM-140292

18. Compagnat M, Mandigout S, Chaparro D, Daviet JC, Salle JY. Validity of the Actigraph GT3x and influence of the sensor positioning for the assessment of active energy expenditure during four activities of daily living in stroke subjects. Clin Rehabil. 2018;32(12):1696-1704. doi:10.1177/02692155187881 16 
19. Talkowski JB, Lenze EJ, Munin MC, Harrison C, Brach JS. Patient participation and physical activity during rehabilitation and future functional outcomes in patients after hip fracture. Arch Phys Med Rehabil. 2009;90(4):618-622. doi:10.1016/j.apmr.2008.10.024

20. Treacy D, Hassett L, Schurr K, Chagpar S, Paul SS, Sherrington C. Validity of different activity monitors to count steps in an inpatient rehabilitation setting. Phys Ther. 2017;97(5):581-588. doi:10.1093/ptj/pzx01 $\underline{0}$

21. Evenson KR, Goto MM, Furberg RD. Systematic review of the validity and reliability of consumerwearable activity trackers. Int J Behav Nutr Phys Act. 2015;12:159. doi:10.1186/s12966-015-0314-1

22. Imboden MT, Nelson MB, Kaminsky LA, Montoye AH. Comparison of four Fitbit and Jawbone activity monitors with a research-grade ActiGraph accelerometer for estimating physical activity and energy expenditure. Br J Sports Med. 2018;52(13):844-850. doi:10.1136/bjsports-2016-0969 90

23. Jones D, Crossley K, Dascombe B, Hart HF, Kemp J. Validity and reliability of the fitbit flex and actigraph gt $3 \mathrm{x}+$ at jogging and running speeds. Int J Sports Phys Ther. 2018;13(5):860-870.

24. Fenton SA, Duda JL, Barrett T. Optimising physical activity engagement during youth sport: a self-determination theory approach. J Sports Sci. 2016;34(19):1874-1884. doi:10.1080/02640414.2016.1 142104

25. Ridley K, Ridgers ND, Salmon J. Criterion validity of the activPAL and ActiGraph for assessing children's sitting and standing time in a school classroom setting. Int J Behav Nutr Phys Act. 2016;13:75. doi:10.1 186/s12966-016-0402-X

26. Greenberg EM, Greenberg ET, Albaugh J, Storey E, Ganley TJ. Rehabilitation practice patterns following anterior cruciate ligament reconstruction: A survey of physical therapists. J Orthop Sports Phys Ther. 2018;48(10):801-811. doi:10.2519/jospt.2018.8264

27. Wright RW, Haas AK, Anderson J, et al. Anterior cruciate ligament reconstruction rehabilitation: Moon guidelines. Sports Health. 2015;7(3):239-243. d oi:10.1177/1941738113517855

28. Rambaud AJM, Ardern CL, Thoreux P, Regnaux JP, Edouard P. Criteria for return for running after anterior cruciate ligament reconstruction: a scoping review. Br J Sports Med. 2018. doi:10.1136/bjsports-20 $\underline{17-098602}$
29. Renner KE, Williams DSB, Queen RM. The reliability and validity of the loadsol((r)) under various walking and running conditions. Sensors (Basel). 2019;19(2). doi:10.3390/s19020265

30. Peebles AT, Maguire LA, Renner KE, Queen RM. Validity and repeatability of single-sensor loadsol insoles during landing. Sensors (Basel). 2018;18(12). $\underline{\mathrm{d}}$ oi:10.3390/s18124082

31. Peebles AT, Ford KR, Taylor JB, Hart JM, Sands LP, Queen RM. Using force sensing insoles to predict kinetic knee symmetry during a stop jump. J Biomech. 2019;95:109293. doi:10.1016/i.jbiomech.2019.07.037

32. Paterno MV, Schmitt LC, Ford KR, et al.

Biomechanical measures during landing and postural stability predict second anterior cruciate ligament injury after anterior cruciate ligament reconstruction and return to sport. Am J Sports Med. 2010;38(10):1968-1978. doi:10.1177/03635465103760 $\underline{53}$

33. Peebles AT, Renner KE, Miller TK, Moskal JT, Queen RM. Associations between distance and loading symmetry during return to sport hop testing. Med Sci Sports Exerc. 2019;51(4):624-629. doi:10.124 9/MSS.0000000000001830

34. Sheerin KR, Besier TF, Reid D, Hume PA. The oneweek and six-month reliability and variability of three-dimensional tibial acceleration in runners. Sports Biomech. 2018;17(4):531-540. doi:10.1080/147 63141.2017.1371214

35. Benjaminse A, Welling W, Otten B, Gokeler A. Novel methods of instruction in ACL injury prevention programs, a systematic review. Phys Ther Sport. 2015;16(2):176-186. doi:10.1016/j.ptsp.2014.0 6.003

36. Shimokochi Y, Shultz SJ. Mechanisms of noncontact anterior cruciate ligament injury. J Athl Train. 2008;43(4):396-408. doi:10.4085/1062-6050-4 $\underline{3.4 .396}$

37. Yu B, Garrett WE. Mechanisms of non-contact ACL injuries. Br J Sports Med. 2007;41 Suppl 1:i47-51. do i:10.1136/bjsm.2007.037192

38. Munro AG, Herrington LC. Between-session reliability of four hop tests and the agility T-test. $J$ Strength Cond Res. 2011;25(5):1470-1477. doi:10.151 9/JSC.0b013e3181d83335 
39. Mann JB, Ivey PA, Mayhew JL, Schumacher RM, Brechue WF. Relationship between agility tests and short sprints: Reliability and smallest worthwhile difference in national collegiate athletic association division-i football players. J Strength Cond Res. 2016;30(4):893-900. doi:10.1519/JSC.0000000000001 $\underline{329}$

40. Brumitt J, Heiderscheit BC, Manske RC, Niemuth P, Mattocks A, Rauh MJ. The lower-extremity functional test and lower-quadrant injury in ncaa division iii athletes: A descriptive and epidemiologic report. J Sport Rehabil. 2016;25(3):219-226. doi:10.11 23/isr.2014-031610.1123/jsr.2014-0316

41. Chmielewski TL, Myer GD, Kauffman D, Tillman SM. Plyometric exercise in the rehabilitation of athletes: physiological responses and clinical application. J Orthop Sports Phys Ther. 2006;36(5):308-319. doi:10.2519/jospt.2006.2013

42. van Grinsven S, van Cingel RE, Holla CJ, van Loon CJ. Evidence-based rehabilitation following anterior cruciate ligament reconstruction. Knee Surg Sports Traumatol Arthrosc. 2010;18(8):1128-1144. doi:10.100 7/s00167-009-1027-2

43. Borges TO, Moreira A, Bacchi R, et al. Validation of the VERT wearable jump monitor device in elite youth volleyball players. Biol Sport. 2017;34(3):239-242. doi:10.5114/biolsport.2017.6600 $\underline{0}$

44. Vlantes TG, Readdy T. Utilizing microsensor technology to quantify match demands in collegiate women's volleyball. J Strength Cond Res. 2017. doi:1 0.1519/ISC.0000000000002208

45. Halson SL. Monitoring training load to understand fatigue in athletes. Sports Med. 2014;44 Suppl 2:S139-S147. doi:10.1007/s40279-014-0253-Z

46. Timpka T, Alonso JM, Jacobsson J, et al. Injury and illness definitions and data collection procedures for use in epidemiological studies in athletics (track and field): Consensus statement. Br J Sports Med. 2014;48(7):483-490. doi:10.1136/bjsports-2013-09324 1

47. Foster C, Florhaug JA, Franklin J, et al. A new approach to monitoring exercise training. J Strength Cond Res. 2001;15(1):109-115.

48. Impellizzeri FM, Rampinini E, Coutts AJ, Sassi A, Marcora SM. Use of RPE-based training load in soccer. Med Sci Sports Exerc. 2004;36(6):1042-1047. doi:10.12 $\underline{49 / 01 . m s s .0000128199 .23901 .2 \mathrm{f}}$
49. Manzi V, Bovenzi A, Castagna C, Sinibaldi Salimei P, Volterrani M, Iellamo F. Training-load distribution in endurance runners: Objective versus subjective assessment. Int J Sports Physiol Perform.

2015;10(8):1023-1028. doi:10.1123/ijspp.2014-0557

50. Watson A, Brickson S, Brooks A, Dunn W. Subjective well-being and training load predict inseason injury and illness risk in female youth soccer players. Br J Sports Med. 2017;51(3):194-199. doi:10.1 136/bjsports-2016-096584

51. Hornsby JH, Green JM, O’Neal EK, Killen LL, McIntosh JR, Coates TE. Influence of terminal RPE on session RPE. J Strength Cond Res.

2013;27(10):2800-2805. doi:10.1519/ISC.0b013e3182 $\underline{830 d 6 c}$

52. Saw AE, Kellmann M, Main LC, Gastin PB. Athlete self-report measures in research and practice: Considerations for the discerning reader and fastidious practitioner. Int J Sports Physiol Perform. 2017;12(Suppl 2):S2127-S2135. doi:10.1123/ijspp.201 6-0395

53. Da Silva DF, Verri SM, Nakamura FY, Machado FA. Longitudinal changes in cardiac autonomic function and aerobic fitness indices in endurance runners: a case study with a high-level team. Eur J Sport Sci. 2014;14(5):443-451. doi:10.1080/17461391.2013.8328 $\underline{02}$

54. Plews DJ, Laursen PB, Kilding AE, Buchheit M. Heart rate variability in elite triathletes, is variation in variability the key to effective training? A case comparison. Eur J Appl Physiol. 2012;112(11):3729-3741. doi:10.1007/s00421-012-23 54-4

55. Saboul D, Balducci P, Millet G, Pialoux V, Hautier C. A pilot study on quantification of training load: The use of HRV in training practice. Eur J Sport Sci. 2016;16(2):172-181. doi:10.1080/17461391.2015.1004 $\underline{373}$

56. Bosquet L, Merkari S, Arvisais D, Aubert AE. Is heart rate a convenient tool to monitor overreaching? A systematic review of the literature. $\mathrm{Br} \mathrm{J}$ Sports Med. 2008;42(9):709-714. doi:10.1136/bjsm.20 $\underline{07.042200}$

57. Daanen HA, Lamberts RP, Kallen VL, Jin A, Van Meeteren NL. A systematic review on heart-rate recovery to monitor changes in training status in athletes. Int J Sports Physiol Perform. 2012;7(3):251-260. doi:10.1123/ijspp.7.3.251 
58. Owen AL, Forsyth JJ, Wong del P, Dellal A, Connelly SP, Chamari K. Heart rate-based training intensity and its impact on injury incidence among elite-level professional soccer players. J Strength Cond Res. 2015;29(6):1705-1712. doi:10.1519/JSC.00000000 00000810

59. Bellenger CR, Fuller JT, Thomson RL, Davison K, Robertson EY, Buckley JD. Monitoring athletic training status through autonomic heart rate regulation: A systematic review and meta-analysis. Sports Med. 2016;46(10):1461-1486. doi:10.1007/s402 79-016-0484-2

60. Buchheit M. Monitoring training status with HR measures: do all roads lead to Rome? Front Physiol. 2014;5:73. doi:10.3389/fphys.2014.00073

61. Flatt AA, Esco MR. Smartphone-derived heartrate variability and training load in a women's soccer team. Int J Sports Physiol Perform. 2015;10(8):994-1000. doi:10.1123/ijspp.2014-0556

62. Plews DJ, Laursen PB, Stanley J, Kilding AE, Buchheit $\mathrm{M}$. Training adaptation and heart rate variability in elite endurance athletes: opening the door to effective monitoring. Sports Med. 2013;43(9):773-781. doi:10.1007/s40279-013-0071-8

63. Nakamura FY, Pereira LA, Esco MR, et al. Intraday and interday reliability of ultra-short-term heart rate variability in rugby union players. J Strength Cond Res. 2017;31(2):548-551. doi:10.1519/ISC.0000000000001 $\underline{514}$

64. Tracey KJ. Physiology and immunology of the cholinergic antiinflammatory pathway. J Clin Invest 2007;117(2):289-296. doi:10.1172/JCI30555

65. Tracey KJ. The inflammatory reflex. Nature. 2002;420(6917):853-859. doi:10.1038/nature01321

66. Pavlov VA, Tracey KJ. The vagus nerve and the inflammatory reflex--linking immunity and metabolism. Nat Rev Endocrinol. 2012;8(12):743-754. doi:10.1038/nrendo.2012.189

67. Aubert AE, Seps B, Beckers F. Heart rate variability in athletes. Sports Med. 2003;33(12):889-919. doi:10.2 165/00007256-200333120-00003

68. Makivic B, Nikic MD, Willis MS. Heart rate variability (hrv) as a tool for diagnostic and monitoring performance in sport and physical activities. Journal of Exercise Physiology. 2013;16(3):103-131.

69. Jose AD, Collison D. The normal range and determinants of the intrinsic heart rate in man. Cardiovasc Res. 1970;4(2):160-167. doi:10.1093/cvr/ $\underline{4.2 .160}$
70. Esco MR, Flatt AA. Ultra-short-term heart rate variability indexes at rest and post-exercise in athletes: evaluating the agreement with accepted recommendations. J Sports Sci Med. 2014;13(3):535.

71. Plews DJ, Scott B, Altini M, Wood M, Kilding AE, Laursen PB. Comparison of heart-rate-variability recording with smartphone photoplethysmography, polar h7 chest strap, and electrocardiography. Int J Sports Physiol Perform. 2017;12(10):1324-1328. doi:1 $\underline{0.1123 / \text { ijspp.2016-0668 }}$

72. Vasconcellos FV, Seabra A, Cunha FA, Montenegro RA, Bouskela E, Farinatti P. Heart rate variability assessment with fingertip photoplethysmography and polar RS800cx as compared with electrocardiography in obese adolescents. Blood Press Monit. 2015;20(6):351-360. doi:10.1097/MBP.0000000000000 143

73. Pernice R, Javorka M, Krohova J, et al. Comparison of short-term heart rate variability indexes evaluated through electrocardiographic and continuous blood pressure monitoring. Med Biol Eng Comput. 2019;57(6):1247-1263. doi:10.1007/s11517-019-0195 7-4

74. Gisselman AS, D’Amico M, Smoliga JM. Optimizing inter-session reliability of heart rate variability - the effects of artefact correction and breathing type. J Strength Cond Res. 2017. doi:10.151 9/JSC.0000000000002258

75. Flatt AA, Esco MR, Nakamura FY. Individual heart rate variability responses to preseason training in high level female soccer players. J Strength Cond Res. 2017;31(2):531-538. doi:10.1519/JSC.0000000000001 482

76. Kemp AH, Koenig J, Thayer JF. From psychological moments to mortality: A multidisciplinary synthesis on heart rate variability spanning the continuum of time. Neurosci Biobehav Rev. 2017;83:547-567. doi:1 $\underline{0.1016 / \text { i.neubiorev.2017.09.006 }}$

77. Thayer JF, Loerbroks A, Sternberg EM. Inflammation and cardiorespiratory control: the role of the vagus nerve. Respir Physiol Neurobiol. 2011;178(3):387-394. doi:10.1016/j.resp.2011.05.016

78. Meeusen R, Duclos M, Foster C, et al. Prevention, diagnosis, and treatment of the overtraining syndrome: joint consensus statement of the European College of Sport Science and the American College of Sports Medicine. Med Sci Sports Exerc. 2013;45(1):186-205. doi:10.1249/MSS.0b013e318279a $\underline{10 \mathrm{a}}$ 
79. Bittencourt NFN, Meeuwisse WH, Mendonca LD, Nettel-Aguirre A, Ocarino JM, Fonseca ST. Complex systems approach for sports injuries: moving from risk factor identification to injury pattern recognition-narrative review and new concept. $\mathrm{Br} \mathrm{J}$ Sports Med. 2016;50(21):1309-1314. doi:10.1136/bjsp orts-2015-095850

80. Flatt AA, Hornikel B, Esco MR. Heart rate variability and psychometric responses to overload and tapering in collegiate sprint-swimmers. J Sci Med Sport. 2017;20(6):606-610. doi:10.1016/j.jsams.2016.1 $\underline{0.017}$
81. Davies GJ, McCarty E, Provencher M, Manske RC. ACL return to sport guidelines and criteria. Curr Rev Musculoskelet Med. 2017;10(3):307-314. doi:10.1007/s 12178-017-9420-9

82. Stanley J, D’Auria S, Buchheit M. Cardiac parasympathetic activity and race performance: an elite triathlete case study. Int J Sports Physiol Perform. 2015;10(4):528-534. doi:10.1123/ijspp.2014-0196 